\title{
NON DESTRUCTIVE TESTING OF ELECTRONIC DEVICES BY ACOUSTIC MICROSCOPY
}

\author{
J. ATTAL and G. CAMBON
}

Centre d'Etudes d'Electronique des Solides $\left({ }^{*}\right)$, U.S.T.L., place E.-Bataillon, 34060 Montpellier Cedex, France

\begin{abstract}
Résumé. - L'intérêt du microscope acoustique précédemment démontré dans le domaine biologique est étendu à des applications microélectroniques. Les propriétés de pénétration et de focalisation des ondes acoustiques dans les solides sont particulièrement bien adaptées à l'observation non destructive de surfaces et sous-surfaces de structures et de composants relevant de la physique des solides tels que circuits intégrés, couches épitaxiées, implantées ou diffusées. A ce jour, c'est l'un des meilleurs moyens de visualiser sous la surface de solides opaques à la lumière et aux électrons.
\end{abstract}

\begin{abstract}
The interest of the scanning acoustic microscope previously demonstrated in the biomedical field is extended to microelectronic applications. The properties of penetrating and focusing of acoustic waves in solids are particularly suitable for non destructive observation of surface and subsurface of solid state structure and device such as integrated circuit, epitaxial, implanted and diffused layers. Up to date it is one of the best ways to visualize underneath the surface of solids opaque to light and electronic rays.
\end{abstract}

1. Introduction. - Developed by C. F. Quate and his collaborators [1-3] at Stanford University the acoustic microscope has now been advanced to the point where it is useful instrument for viewing microscopic objects sized larger than $1 \mu \mathrm{m}$. For several years biological specimens has been the widest scope of applications. Since the microscope was operating by reflection the interest of this instrument turned to solid state application. Indeed, in most cases these materials which are completely opaque optically can be readily penetrated by an acoustic wave. With this instrument detail lying beneath the surface of such samples could be revealed. This is of the most importance for application to the problem of non destructive testing in a microscopic scale. During these last few years we have been concerned in developing this instrument in microelectronics. In that field, acoustic microscopy is particularly suitable for observation of defects, structures or stresses inside semiconductors, metals and devices such as integrated circuits. This can be microcracks, voids, grain boundaries or also change of structure or cristalline phase of an element, a compound or an alloy. Defects under coatings, ion implanted region, epitaxial layers are also good candidates for acoustic microscopy examination.

The purpose of this paper is to bring out the most important feature of this new microscope which is imaging under surfaces, then we will discuss on some of numerous applications in the field of non destructive testing.

2. Acoustic microscope configuration. - Figure 1 gives a schematic configuration of the microscope

(*) Associé au C.N.R.S.

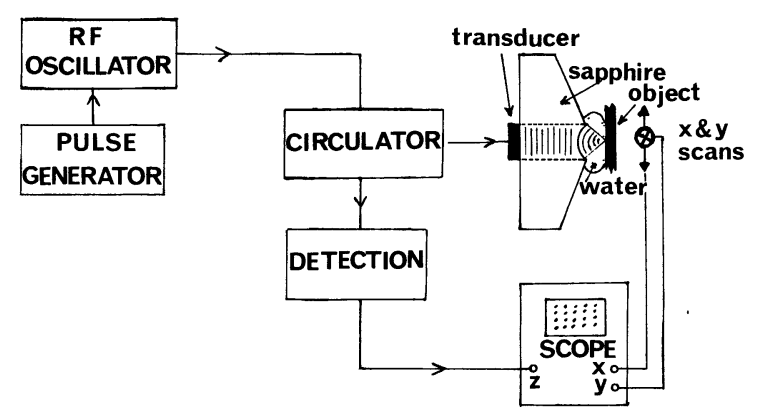

FIG. 1. - Schematic configuration of the acoustic microscope operating in reflection mode.

operating in reflection mode. A collimated acoustic wave is generated with a zinc oxyde thin film transducer at $600 \mathrm{MHz}$. The acoustic beam is focused down to the sample through a spherical lens constituted by a sapphire-water interface. The size of the acoustic beam in water at the focal point is of the order of $1 \mu \mathrm{m}$. The wave reflected by the sample is collected back to the lense which recollimates the beam before detection by the transducer. To get rid of spurious reflection inside the sapphire and to prevent the reflected signal from mixing to the incident wave we are operating in pulse mode.

An original technique of detection give the amplitude and the phase of the reflected wave with a fairly good signal to noise ratio. The output signal serves to modulate the intensity of the electron beam of an imaging scope.

The object is mechanically scanned along two orthogonal directions in the focal plane of the lens in such a way that the acoustic reflection coming from 
the sample is recorded point by point. The precision and repeatability exceed the limit set by the resolution of the lens. The fast scan ( 30 to $100 \mathrm{~Hz})$ is obtained from the movement of a vibrator which gives a maximum amplitude peak to peak of 5 millimeters. The slow can $(0.1$ to $1 \mathrm{~Hz})$ is assured by a micromotor which lifts up and down the total assembly. Inductive pick-up coils are used to display the line scans on the imaging scope in synchronism with the object, by transducing the $X$ and $Y$ positions.

3. Resolution performance. - In figure 2 we show an acoustic reflection micrograph of a part of an integrated circuit (Fig. 2b) compared with a scanning

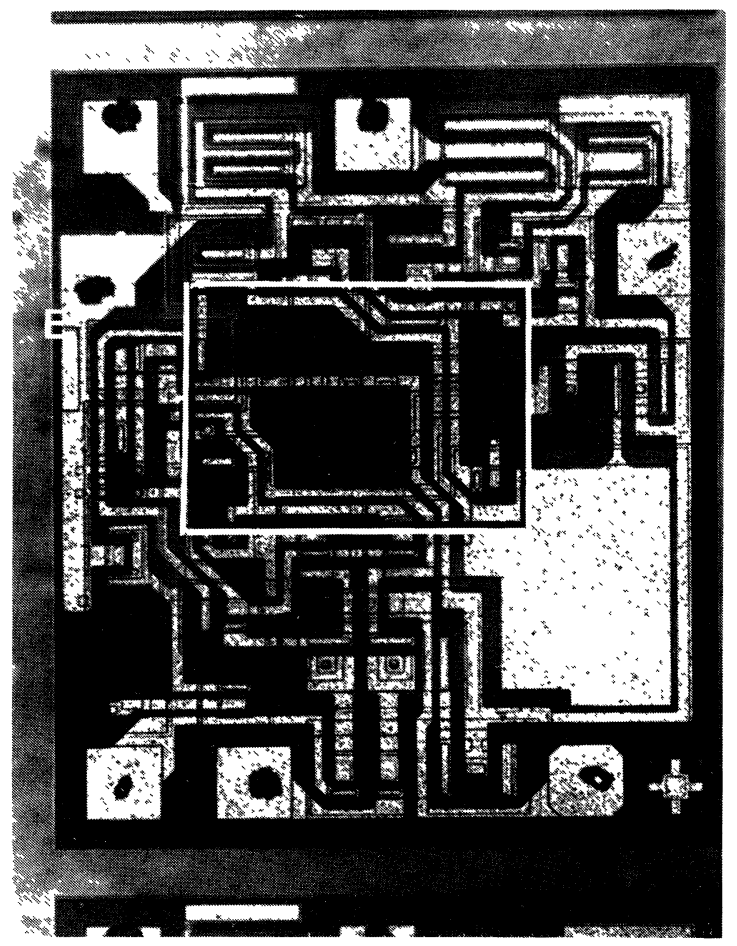

a)

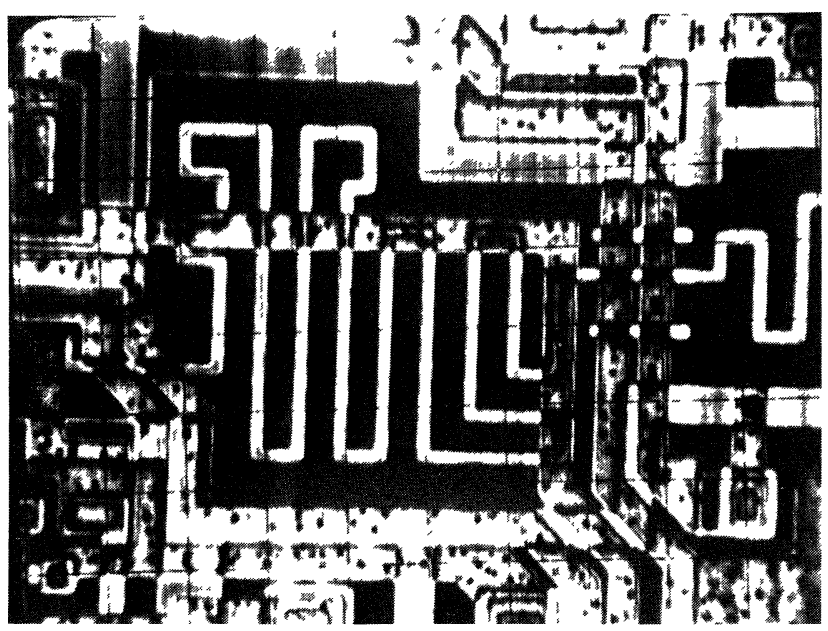

b)

FIG. 2. - Scanning secondary electron $(a)$ and acoustic $(b)$ micrographs of a part of the integrated circuit SFC 2741 supplied by Sescosem. secondary electron image of the whole circuit (Fig. 2a). The acoustic photo is made at an acoustic frequency of $600 \mathrm{MHz}$. We note that the contrast of the acoustic picture is much higher than that of the electron picture. Even more interesting is the fact that the

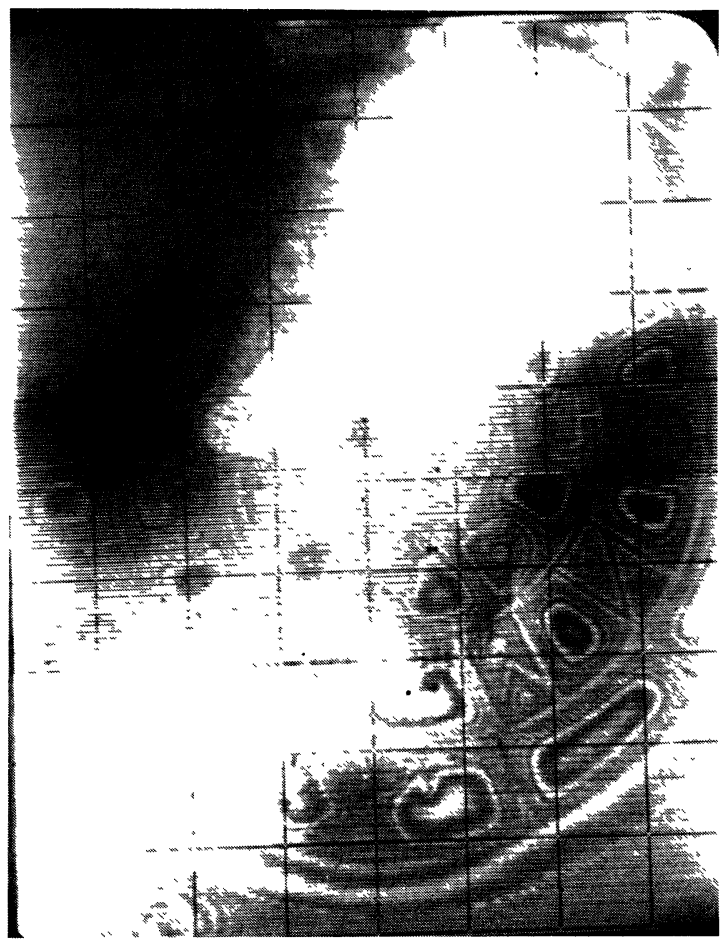

a)

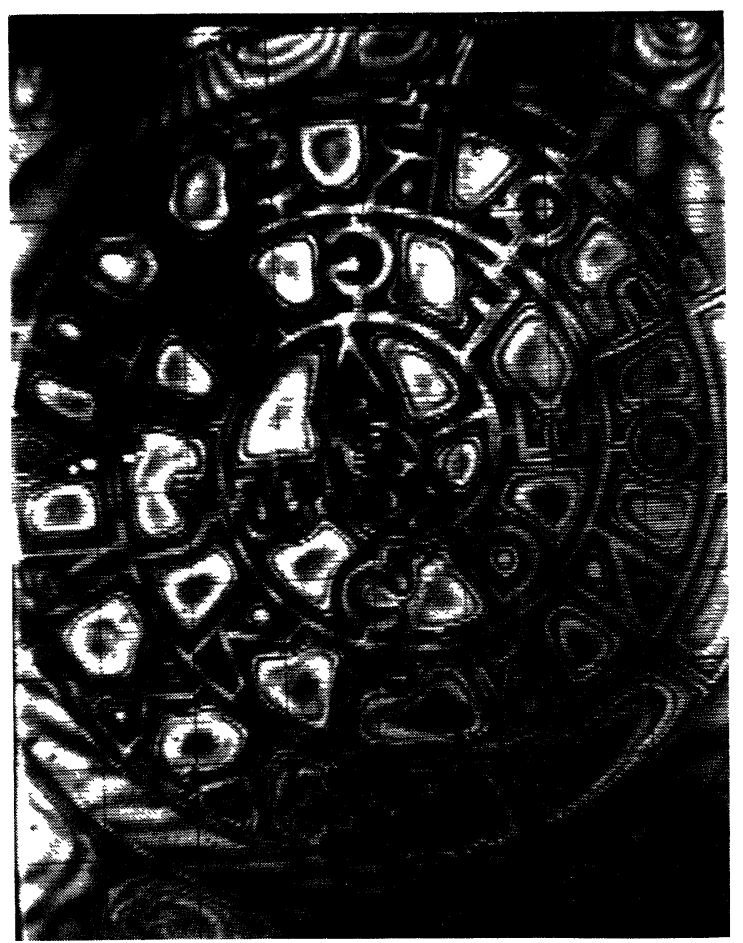

b)

Fig. 3. - Acoustic image of the test object for two different focusings inside the sample : $a$ ) focusing is onto the surface; b) focusing is $12.5 \mu \mathrm{m}$ inside the object. 
various defects are visible in the acoustic image. Naturally, it is difficult to give a thorough explanation of the complexity of such a circuit constituted with diffused layers and several coatings superimposed. Our understanding is not yet extended to the point where we can give accurate informations to the manufacturer.

4. Exploration of subsurface. - In order to show up the microscope's unique capability we have realized a test object constituted with three different materials : an electron microscopy grid is glued on a half mil thickness sheet of mylar coated with a few thousand angstroms of aluminium in such a way that the grid and the coating are on each side of the mylar. Optically viewed from the coated side the object is completely opaque and the surface is perfectly smooth. In figure 3 we show the acoustic images when focusing on the aluminium coating and on the grid through aluminium and mylar. It is clearly evident that we are able to have a sharp focusing inside the object in spite of the large acoustic reflection which occurs between water aluminium and mylar. When we focus onto the aluminium coating the grid is blurred and large dark and bright areas of interference patterns are superimposed to the image.

With this simple example we have demonstrated the important feature of the acoustic microscope. For a better understanding of viewing the interior of an object we have to introduce two parameters characterizing this ability.

The first one is the depth of field which is the maximum distance between two points on the axis which are simultaneously in focus. A simple calculation derived from optics gives its expression $\rho$ as a function of the wavelength and the aperture of the lens :

$$
\rho \simeq \frac{2 i}{\sin ^{2} \theta_{\mathrm{m}}}
$$

For example at $600 \mathrm{MHz}$ in water with a lense aperture of $60^{\circ}, \rho$ is of the order of $7.5 \mu \mathrm{m}$. Inside the object this expression is rather complicated as it depends on the nature and the position of the object in respect with the center of curvature of the lens. This leads us to introduce the second parameter which is the penetration of the focused beam. As we see in figure 4 it is dependent of the distance between the position of the interface sample-liquid and in first approximation is given by the linear relation :

$$
d^{\prime}=\frac{d}{n}
$$

where $d$ and $d^{\prime}$ are the distance between interface water-object and focus (virtual and actual) $n$ is the acoustic index of refraction defined as the ratio of acoustic velocities between object and water. For high value of $n$ (that is for most of solids) the acoustic beam

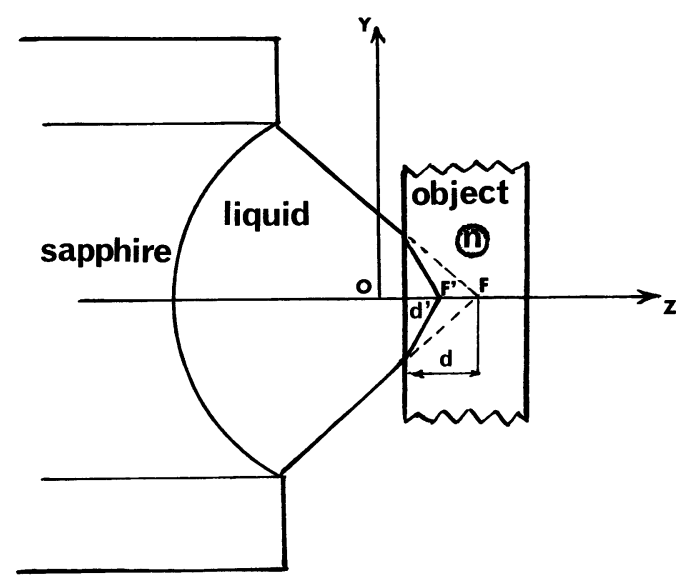

FIG. 4. - Geometry for the ray tracing inside an object.

strongly converges into a point near the interface and the maximum depth of penetration turns around ten to twenty microns.

5. Typical applications. - To illustrate some of the numerous applications we first present acoustic micrographs of an epitaxial layer of $\mathrm{Ga}_{1-x} \mathrm{Al}_{x} \mathrm{Sb}$ on a $\mathrm{Zn}$ Te substrate $(x=10$ per cent). Figure $5 a$ shows the acoustic image of the surface of such a device which is relatively smooth with some pin holes. When focusing down to the substrate at $3.5 \mu \mathrm{m}$ from the surface, figure $5 b$, we see gallium inclusions (white spots) and trails probably due to the substrate polishing. These inclusions of gallium have been revealed elsewhere [4] using a Castaing's microsonde. On the contrary no other information has been obtained on the origin of the trails. To monitor this change we have recorded on figure $5 c$ the detected output signal for one line scan at different positions inside the object. This clearly shows up the transitions between the surface (top trace) and the interior of the substrate (bottom trace) with a maximum of detail at the interface.

An other interesting problem is the imaging of an ion implanted samples of semiconductor. The material used is quartz partially implanted with ions $4 \mathrm{He}^{+}$. Because of the small change in amplitude between implanted and non implanted regions we have devised a phase measurement system. This technique can give informations about the sound velocity variations from point to point. Details much less than the wavelength can be resolved. This technique is comparable to phase contrast in optical microscopy; the phase of the reflected signal is continuously compared to a reference phase coming from the electromagnetic input signal and the resulting signal proportional to the cosine of the phase serves to modulate the light intensity of the imaging scope.

Figure 6 shows the recording output of the cosine of the phase corresponding to one $\mathrm{X}$ line scan and brings 


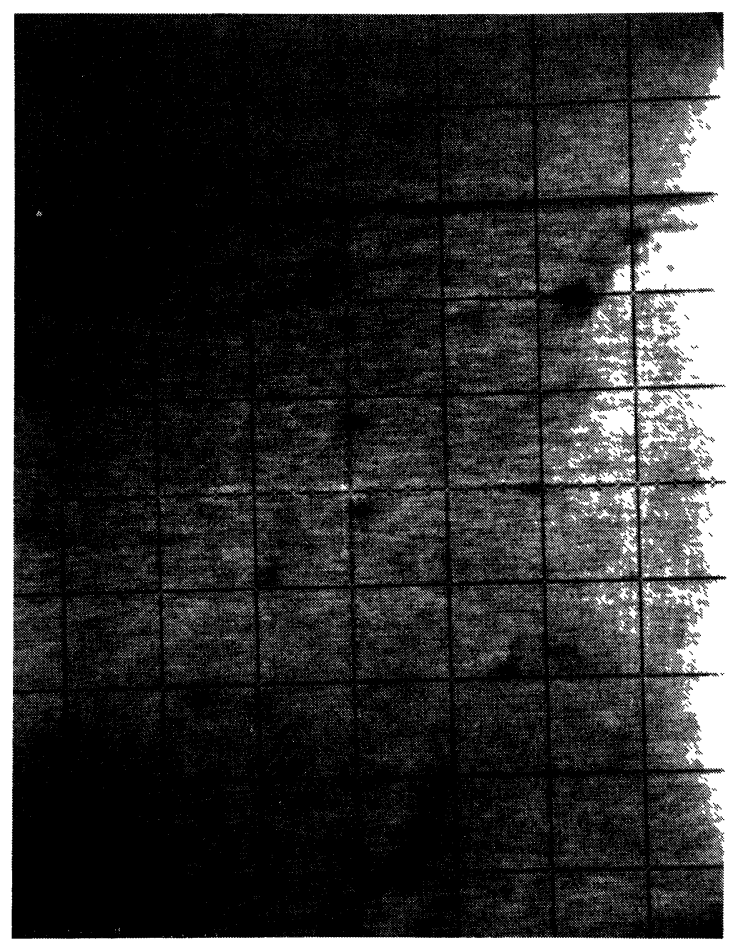

a)

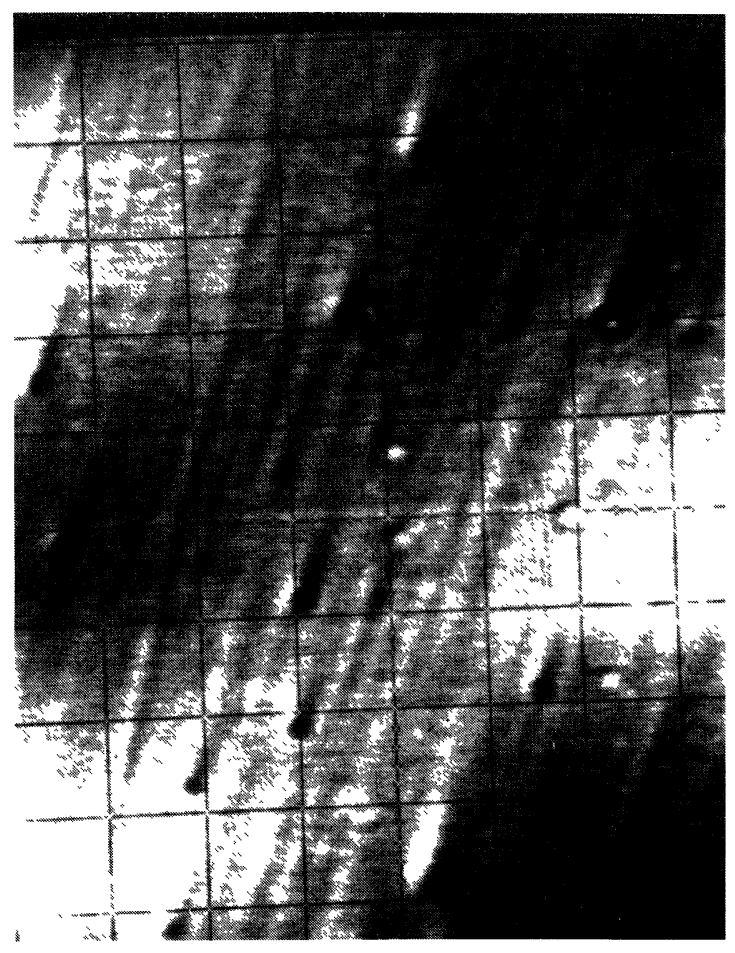

b)

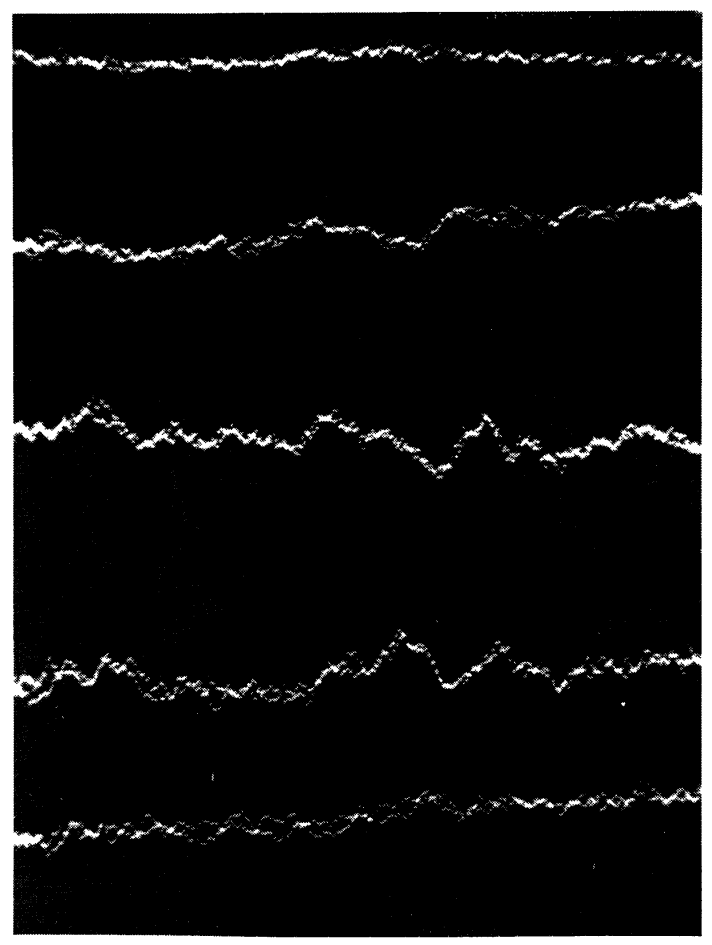

c)

FIG. 5. - Acoustic examination of an epitaxial layer of $\mathrm{Ga}_{1-x} \mathrm{Al}_{x} \mathrm{Sb}$ on a $\mathrm{ZnTe}$ substrate $(x=10$ per cent). Scale $12 \mu \mathrm{m}$ per division. a) focusing on the surface ; $b$ ) focusing down to the substrate ; $c$ ) recordings of one line scan for different focusings from the surface (top trace) to the interior of the substrate (botton trace). The maximum of structure corresponds to the interface. 


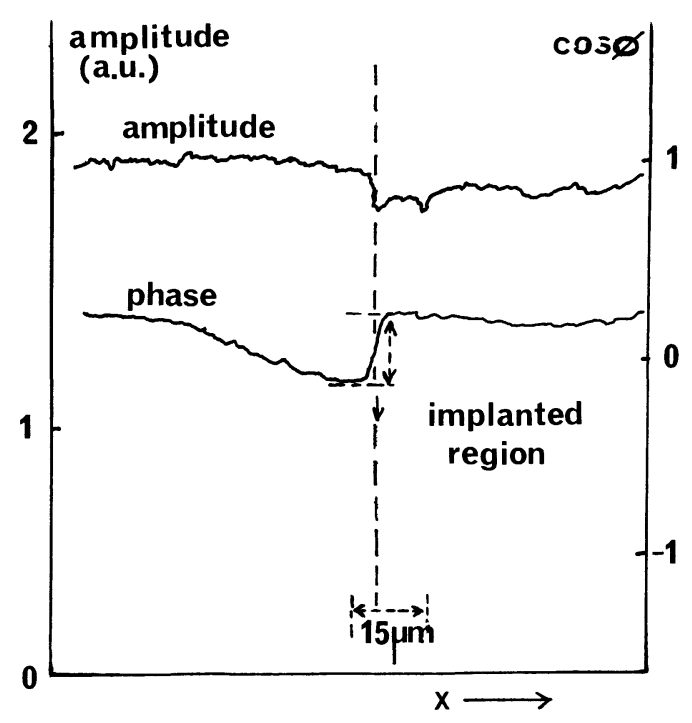

FIG. 6. - Variations of the amplitude and phase of the reflected signal for one line scan. The sample is a quartz implanted with ions $4 \mathrm{He}^{+}$.

out a jump of the phase between implanted and non implanted region. This jump is equal to $\pi / 8$ and reveals the existence of a step of $780 \AA$ caused by an expansion of the implanted region. The change of phase in figure 6 is not a purely topographic effect because the phase information takes into account the travel of the acoustic wave inside the sapphire-water medium and the reflectivity of the scanned object. Nevertheless this change of reflectivity between implanted and non implanted regions is negligible compared to the travel delays of the acoustic wave. On the contrary direct measurements of the amplitude (Fig. 6 top trace) give the variations of the reflectivity between the two regions. We have not yet given an interpretation of these results which seem to be very promising. We shall only note that the height of the step is in good agreement with the data of the manufacturer [5] (measured by Talystep method). With this technique we should be able to appreciate a variation of phase of about $\pi / 30$.

6. Conclusion. - We have clearly shown the particular interest of the acoustic microscope which can give an image of structure underlying the surface of material optically opaque. This makes that this instrument has no equivalent among the microscopes using other type of radiations and is particularly suitable for microelectronic applications. As a consequence, acoustic microscopy does not require destructive sample preparation, and in contrast with electron microscopy does not degrade the structure of the objects during acoustic irradiation.

\section{References}

[1] Lemons, R. A. and Quate, C. F., Appl. Phys. Lett. 24 (1974) 163. [2] Atalar, A., Quate, C. F. and Wickramasinghe, H. K., Appl. Phys. Lett. 31 (1977) 791.
[3] Lemons, R. A. and Quate, C. F., Appl. Phys. Lett. 25 (1974) 251.

[4] Gautier, P., Thèse de $3^{\mathrm{e}}$ Cycle (1978).

[5] Hartemann, P., Revue Phys. Appl. 12 (1977) 843. 